\title{
Miniaturized Spectral Imaging Instrumentation for Planetary Exploration
}

\author{
Näsilä, Antti
}

IEEE

2020-03

Näsilä , A \& Kohout, T 2020 , Miniaturized Spectral Imaging Instrumentation for Planetary Exploration . in 2020 IEEE Aerospace Conference : AERO 2020 . , 9172437 , IEEE

Aerospace Conference Proceedings, IEEE , 2020 IEEE Aerospace Conference, Big Sky , United States , 07/03/2020 . https://doi.org/10.1109/AERO47225.2020.9172437

http://hdl.handle.net/10138/339183

https://doi.org/10.1109/AERO47225.2020.9172437

unspecified

acceptedVersion

Downloaded from Helda, University of Helsinki institutional repository.

This is an electronic reprint of the original article.

This reprint may differ from the original in pagination and typographic detail.

Please cite the original version. 


\section{Miniaturized Spectral Imaging Instrumentation for Planetary Exploration}

\author{
Antti Näsilä \\ VTT Technical Research Centre of Finland Ltd \\ Tietotie 3, 02150 Espoo \\ Finland \\ antti.nasila@vtt.fi
}

\author{
Tomas Kohout \\ Department of Geosciences and Geography \\ P.O. Box 64, FI-00014 \\ University of Helsinki, Finland \\ tomas.kohout@helsinki.fi
}

\begin{abstract}
In this paper, we introduce a novel concept for miniaturized spectral imaging instrument which can be used for different planetary exploration missions. The instrument design utilizes a modular approach, so it is easily tailored for different mission profiles. It can be fitted in ca. 1 CubeSat unit, thus making it suitable for all CubeSat sizes from $2 \mathrm{U}$ upwards. Depending on the mission requirements, the instrument can perform measurements from $0.5 \mu \mathrm{m}$ up to $4 \mu \mathrm{m}$. The visible and near-infrared $(0.5 \mu \mathrm{m}-1.6 \mu \mathrm{m})$ components have already been flown and validated in low Earth orbit.
\end{abstract}

\section{TABLE OF CONTENTS}

1. INTRODUCTION

2. SCIENTIFIC RATIONALE 1

4. MISSION EXAMPLES . .1

5. DEMONSTRATOR FLIGHT RESULTS......................5 6. SUMMARY AND CONCLUSIONS ..........................6 REFERENCES . .7

BIOGRAPHY . .7

\section{INTRODUCTION}

Nanosatellite instrumentation has been developing rapidly during the last decade. Together with the European Space Agency (ESA), VTT started developing nanosatellite compatible hyperspectral imaging technologies already in 2012 and the first demonstrator was launched in summer 2017 on-board the Finnish Aalto-1 nanosatellite. This was the first imager on a nanosatellite capable of hyperspectral imaging. Alongside this activity, VTT also developed a similar spectral imager for atmospheric measurements utilizing solar occultation.

In 2015 the development was focused to planetary exploration, when VTT started developing the first version of the Asteroid Spectral Imager ASPECT for ESA's Asteroid Impact Mission (AIM, now called Hera) [1, 2]. The imager was planned to be flown on a $3 \mathrm{U}$ cubesat, which would be released around the binary asteroid Didymos. For this purpose it was necessary to add measurement capability in the infrared and at the same time design towards more modular instrument concept, which could be easily tailored to different applications. The first demonstrator for the near infrared spectral imager was successfully tested and verified in LEO during the winter 2018 - 2019 onboard the Reaktor Hello World nanosatellite.

The capability to combine different wavelength ranges in a compact volume makes the instrument highly useful in modern planetary exploration missions. Many larger space missions are planning to use smaller secondary spacecraft (InSight, Hera) to perform secondary functions or to address different science objectives. As the larger missions are targeting very different objects (inner planets, outer planets, asteroids, comets) the required instrumentation suite will be different. With standard CubeSat platforms and our modular instrumentation approach, it is easy and fast to tailor an instrument suite for all types of targets and science cases, ranging from geological measurements to atmospheric science.

\section{SCIENTIFIC RATIONALE}

Planetology

Knowledge of planetary compositions is of a key importance in planetology. It enables us to determine the origin of solid material in different zones of our Solar System, as well as to understand current processes such as space weathering (interaction of the planetary surfaces with solar wind, dusty particle impacts, or galactic radiation) or planetary collisions (impacts).

Reflectance spectroscopy is one of the widely used tools to determine the composition of planetary surfaces. The key diagnostic features in the planetary spectra are $\mathrm{Fe}^{2+}(\sim 1$ and $2 \mu \mathrm{m})$ and $\mathrm{Fe}^{3+}$ absorptions $(\sim 0.7 \mu \mathrm{m})$ in silicates, $\mathrm{H}_{2} \mathrm{O}$ and $-\mathrm{OH}$ features $(\sim 1.4,1.9$ or $2.7-3 \mu \mathrm{m}), \mathrm{C}-\mathrm{H}$ bonds in organics $(\sim$ 3-3.6 $\mu \mathrm{m}), \quad \mathrm{Si}-\mathrm{O}$ silicate vibration bands in visible - near-infrared (VIS-NIR), or various ices and gases in mid-infrared (MIR).

Hyperspectral imaging enables spatial resolution of these features, and thus mapping of composition of planetary surfaces in order to determine homogeneity of the target body or distribution of various minerals or volatile compounds. The continuous spectra measurement over 
broad wavelength enables determination of spectral continuum. The shape of the continuum is essential in determination of surface grain size, or in detection of the space weathering or shock effects.

\section{Atmospheric science}

Another important topic is the abundance of gases or substances in planetary atmospheres. Capability to identify different gas species can bring insight to the evolution of the planetary body and to the processes related to the climate.

One of the most useful techniques for performing atmospheric measurements from a small spacecraft is solar occultation [3]. In this method, the instruments look directly at the Sun during sunsets and sunrises. There are two great benefits: the Sun is the most powerful source of light in our solar system, and the method is self-calibrating as the measurement signal is always divided by the out-ofatmosphere signal. These are very useful characteristics when utilizing a small measurement platform.

With this technique, it is possible to identify many gases in the atmosphere based on their absorption spectra and at the same time the vertical profiles can also be generated.

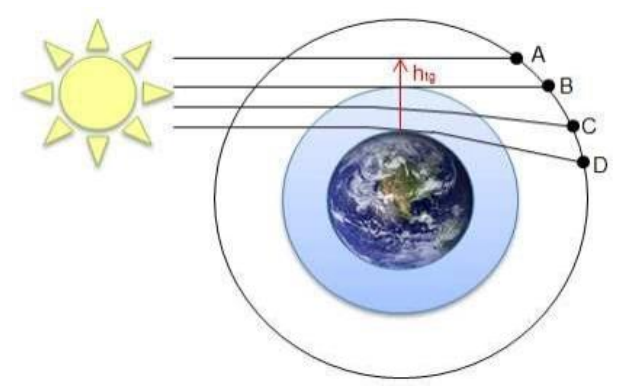

Figure 1. Principle of solar occultation measurement. The first measurement A measures the out-ofatmosphere signal, while the other measurements (B-C) measure the atmospheric absorption at different altitudes.[3]

\section{Instrument Concept}

\section{Principle of operation}

In contrast to more traditional spatial scanning imaging spectrometers, VTT's spectral imager takes 2D snapshots at a given wavelength. When multiple snapshots are combined, a spectral datacube is formed, where the wavelength bands are separated in the time domain [4]. One of the key benefits for this type of spectral imager is that the wavelengths are software programmable, and the measurement sequences can be adjusted in-flight. The downside is that the spectral bands are recorded at different times, which in some cases can be problematic with fast moving platforms. However in many cases, like the ones described in Chapter 4, this is not a problem as the measurement time is fast when compared to the movement and/or there is sufficient tracking capability.
Tunable Fabry-Perot Interferometer [5,6]

Key component in the spectral imager is a tunable FabryPerot Interferometer (FPI) developed at VTT. The FPI consists of two parallel reflective surfaces, which causes the beams of light to reflect back and forth between the mirrors, creating a constructive interference within the interferometer at certain wavelengths. This allows some wavelengths to be transmitted while others are reflected away, thus the interferometer can be used as a bandpass filter. The theoretical wavelength of the transmission bands is determined by

$$
\mathrm{m} \lambda=2 \mathrm{~d} \cos (\theta)
$$

where $\mathrm{m}$ is the interference order, $\lambda$ is the transmitted wavelength, $\mathrm{d}$ is the distance between the surfaces and $\theta$ is the angle of incidence. In practice, the transmitted wavelength does not exactly follow this relation due to nonidealities of the mirrors, but the behavior is similar. The angle dependence needs to be taken into account in optics design. The most common solution is to collimate the light through the filter, so there is no large variation in the ray angles. Non-ideal collimation causes a wavelength shift across the image (spectral smile), i.e. the wavelength in the corner is slightly different than in the center, but the design can be made in a way that the maximum shift is less than 1$2 \mathrm{~nm}$, which is enough for most applications.

When using the filter as a tunable bandpass filter, the operational range is limited by the free spectral range (FSR), i.e. the separation of the consecutive interference peaks. When operating at low orders of interference, the FSR is wide enough for most applications, as shown below.

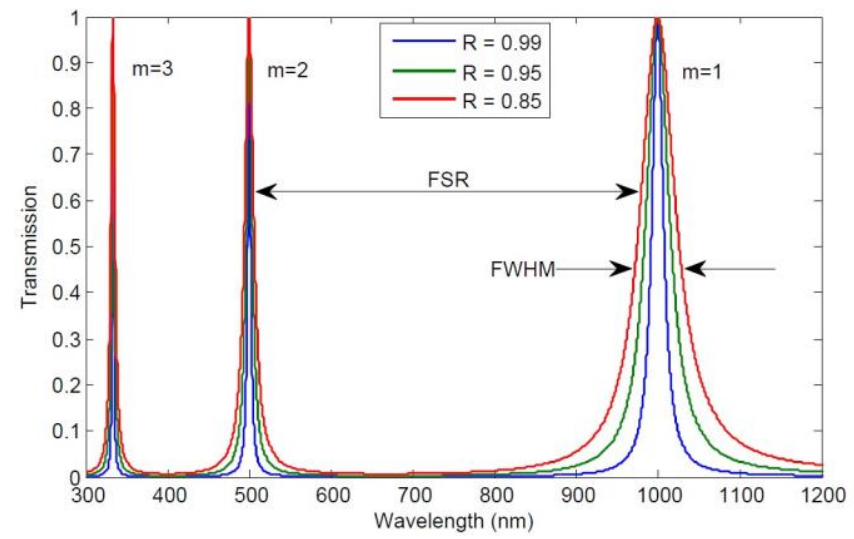

Figure 2. Theoretical example of FPI transmission at different mirror separations. At each gap, there is only one transmission peak, thus the FPI acts as bandpass filter.

The full-width-at-half-maximum (FWHM) of the peaks is determined by the quality of the mirrors (reflectivity) used in the interferometer. Higher reflectivity creates narrower peaks and lower reflectivity broadens the peaks. Another factor affecting the FWHM is the order of interference, higher interference orders create narrower transmission peaks. 
In order to achieve the high reflectivity, dielectric Bragg reflectors are often used. This type of reflector is made by stacking materials with high and low refractive indices in layers that have thickness of quarter of a wavelength $(\lambda / 4)$. This type of reflector has a very high reflectivity, but rather narrow operational range. Another characteristic of this type of mirror is that it is highly reflective only at the optimization wavelength, and transmissive outside the operational range. With these kinds of mirrors, it is possible to achieve FWHM's of 1-5 nm, even at low interference orders.

In many cases extremely high reflectivity is not required. In these cases, the mirrors can be made simply with a metallic coating, e.g. silver. The benefit of this is the broad operational range, as silver is reflective over very broad wavelength range. With these types of filters, the FWHM's of 10-30 nm are achieved at low interference orders.

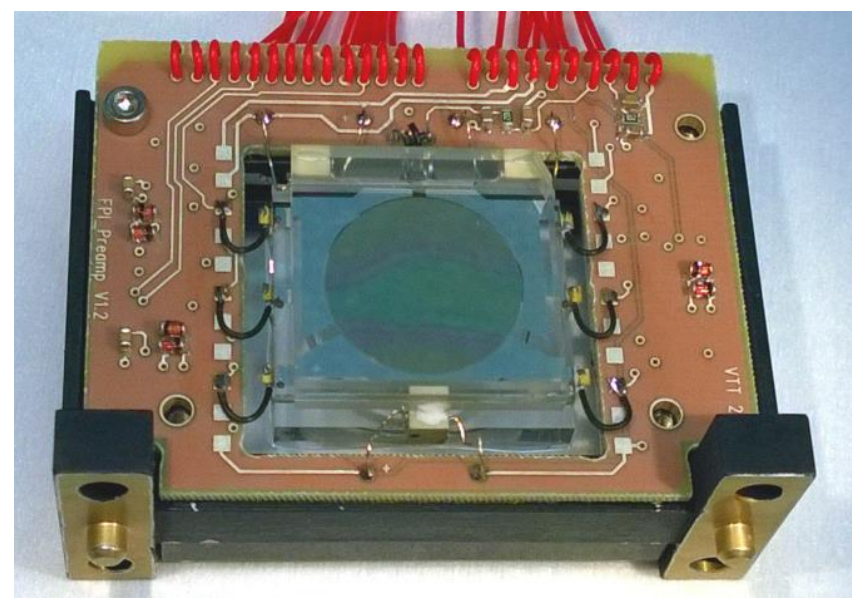

Figure 3. FPI module of the Aalto-1 Spectral Imager flight model.

The gap and parallelism of the FPI is controlled in a closed loop. There are three capacitance measurement electrodes placed around the optical aperture, which are used to measure the distance between the mirrors. These capacitance measurements are then coupled to three piezo actuators, which control the physical gap. [4]

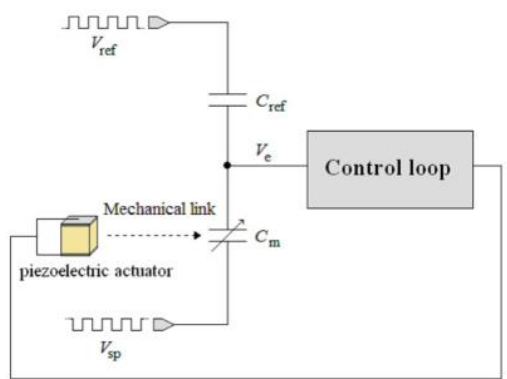

Figure 4. Control principle for the FPI. Only one measurement/actuator channel is drawn.

\section{Optical design}

Optical design for an FPI-based spectral imager is very simple and robust. Front optics are used to collimate the beam through the filter and rear optics focus the light on the detector. The same optical concept can be used for all applications with minor modifications, i.e. adjusting the focal length. The same concept also works with both imaging and non-imaging detectors.

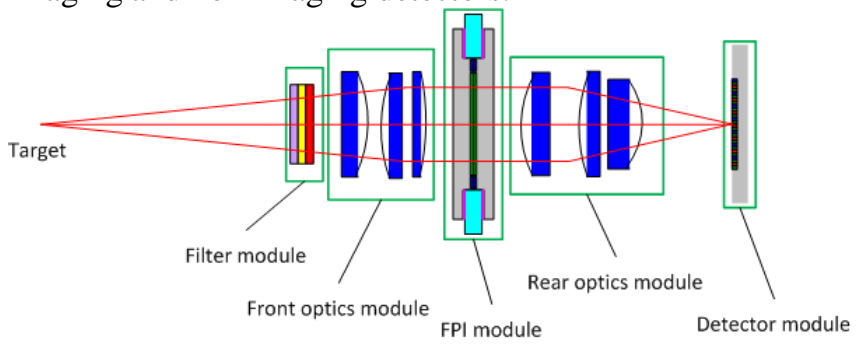

Figure 5. Optical design for FPI based spectral imager. Front optics collimate the light beam through the FPI and the rear optics focus the image on the detector. Filter module in front houses the long and shortpass filters which define the operational wavelength range.

\section{Electrical design}

The functional blocks of the spectral imager are the FPI controller, High voltage generator for the piezo actuators, microcontroller for handling the communications, the detector, readout electronics (usually an FPGA), a buffer memory and a watchdog which can alert the spacecraft computer in case of malfunction.

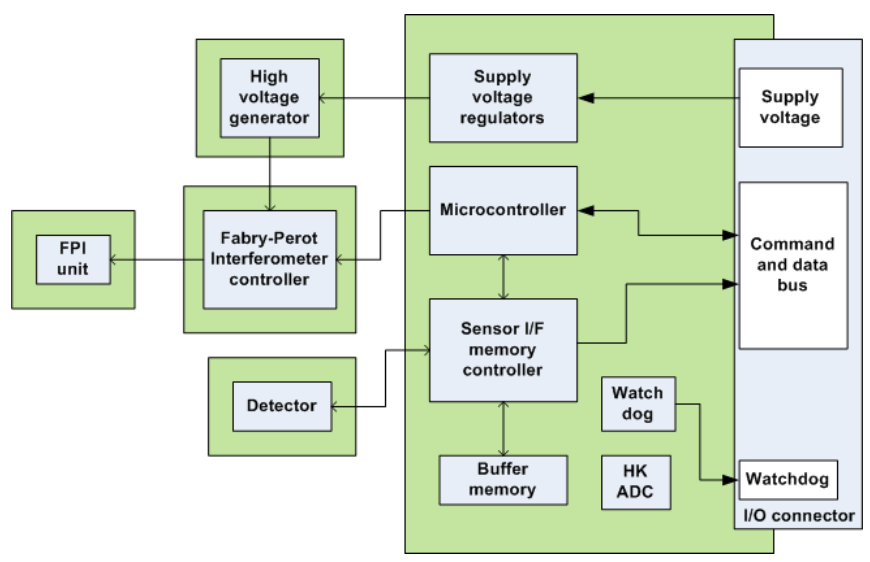

Figure 6. Electrical block diagram of the spectral imager.

The most delicate part of the electronics is the FPI controller, which needs to control the FPI mirrors with subnanometer precision.

The FPI gap is controlled by measuring the capacitance between electrodes deposited on the mirror substrates. The gap is adjusted by three piezo-electric actuators mounted between the FPI mirrors. The gap is measured as the 
capacitances between three sets of electrodes positioned around the FPI mirrors.

The capacitances are measured using a half-bridge configuration (Figure 4). The half-bridge consists of a reference capacitor, $\mathrm{C}_{\text {ref }}$, and the changing capacitance between the FPI mirrors, $\mathrm{C}_{\mathrm{m}}$. The bridge is fed by two voltages, $V_{\text {ref }}$ and $V_{\text {sp. }} V_{\text {ref }}$ is constant and common for all three control channels. The voltage $\mathrm{V}_{\mathrm{sp}}$ is set by a digital-toanalogue converter and represents the set-point of the control loop. Each channel has its own DAC and $\mathrm{V}_{\text {sp. The }}$ reference and set-point voltages both have a square wave form and their phases are opposite.

The voltage $\mathrm{V}_{\mathrm{e}}$ represents the imbalance or error voltage which differs from zero if the balance condition $\mathrm{V}_{\text {ref }} \mathrm{C}_{\text {ref }}=$ $\mathrm{V}_{\mathrm{sp}} \mathrm{C}_{\mathrm{m}}$ is not met. This error signal is amplified, demodulated in a phase-sensitive detector, and fed to the FPI controller and further via high voltage converters to the piezo-electric actuators directly affecting the FPI gap and thus the capacitances $C_{m}$. The reference capacitors and preamplifiers are placed on a PCB surrounding the FPI in order to keep the size of the high-impedance nodes as small as possible.

The bridge imbalance signals from the proximity electronics are demodulated from the $1 \mathrm{MHz}$ carrier and the resulting signals act as error signals for the gap controllers. The controllers are implemented as analogue PI-controllers. These controllers feed the high-voltage amplifiers which supply $0-90 \mathrm{~V}$ to the piezo-electric actuators. A transformer coupled boost converter generates the piezo amplifier supply voltage.

\section{MISSION EXAMPLES}

\section{APEX CubeSat for Hera mission [1, 2, 7, 8]}

The first mission for the modular spectral imager is European Space Agency's Hera mission. Part of this mission is the Asteroid Prospection Explorer CubeSat APEX, which has the Asteroid Spectral Imager ASPECT as one of the payloads. Planned launch date for Hera and APEX is in 2024.

Hera will study binary asteroid Didymos (primary $780 \mathrm{~m}$ and secondary $170 \mathrm{~m}$ in size) - target of the NASA DART (Double-Asteroid Redirection Test) kinetic impactor test mission. DART will impact the secondary asteroid, change its orbiting period, and create approx. 2-4 m sized crater with associated ejecta. APEX with its VIS-NIR hyperspectral imager will map in detail the composition of both asteroids, distribution of impact ejecta, space weathering effects on the asteroid surface, and impactinduced changes in the composition of the crater material.

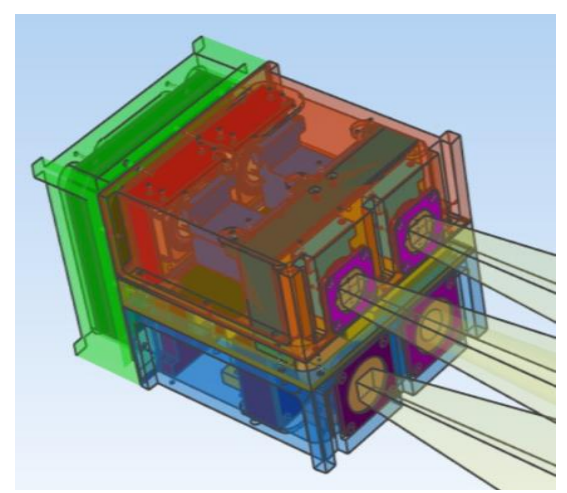

Figure 7. 3D rendering of the ASPECT instrument.

ASPECT has four measurement channels: VIS (500 - 900 $\mathrm{nm})$, NIR1 (850 - $1275 \mathrm{~nm})$, NIR2 (1225 - $1650 \mathrm{~nm})$ and SWIR (1600 - $2500 \mathrm{~nm})$. VIS and NIR channels have imaging capability, while the SWIR channel is a single point spectrometer. All channels have good overlap with each other, so it is possible to construct the complete spectral slope from $500 \mathrm{~nm}$ up to $2500 \mathrm{~nm}$.

Table 1. Key parameters for ASPECT.

\begin{tabular}{|l|l|l|l|l|}
\hline Parameter & VIS & NIR1 & NIR2 & SWIR \\
\hline FoV [deg] & $10^{\circ} \times 10^{\circ}$ & $6.7^{\circ} \times 5.4^{\circ}$ & $6.7^{\circ} \times 5.4^{\circ}$ & $5^{\circ}$ circular \\
\hline $\begin{array}{l}\text { Spectral } \\
\text { range [nm] }\end{array}$ & $500-900$ & $850-1275$ & $1225-1650$ & $1600-2500$ \\
\hline $\begin{array}{l}\text { Image size } \\
\text { [pixels] }\end{array}$ & $1024 \times 1024$ & $640 \times 512$ & $640 \times 512$ & 1 pixel \\
\hline $\begin{array}{l}\text { No. spectral } \\
\text { bands }\end{array}$ & ca. 14 & ca. 14 & ca. 14 & ca. 30 \\
\hline $\begin{array}{l}\text { Spectral } \\
\text { resolution } \\
\text { [nm] }\end{array}$ & $<20$ & $<40$ & $<40$ & $<40$ \\
\hline
\end{tabular}

\section{Comet Interceptor [9]}

Comet Interceptor is a newly selected ESA F-mission to a dynamically new comet from Oort cloud. One of the key payloads is a modular hyperspectral imaging system MIRMIS (Modular Infrared Molecules and Ices Sensor) to detect the mineral, ice, and gas compounds in the comet nucleus and coma in NIR-MIR range, as well as to map temperature of the nucleus. The mission is first-of-its-kind to study the pristine material from the outer Solar System. The planned launch date for Comet Interceptor is in 2028.

Two of the MIRMIS channels, NIR and MIR, are based on VTT's modular instrument concept. The NIR channel, measuring at ca. 1-1.6 $\mu \mathrm{m}$ has the same design as the NIR channels used for APEX. The MIR channel is a new development, and it will measure from $2.5 \mu \mathrm{m}$ up to $7 \mu \mathrm{m}$. The MIRMIS MIR channel exploits the fact that FPI's made with Bragg reflectors are transmissive outside their operational range. This allows several filters to be stacked in series which enables wider operational range (Figure 8). 


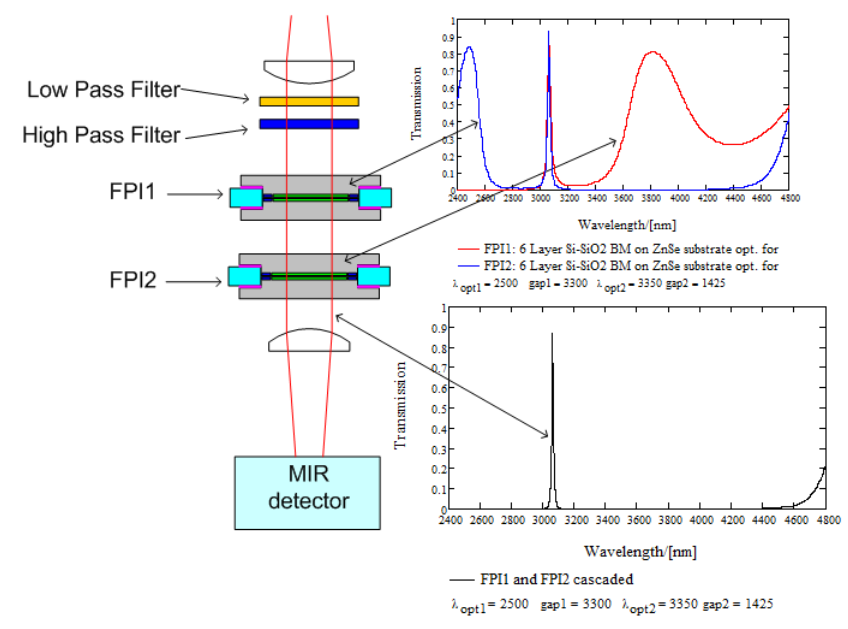

FPI1 and FPI2 Fabry-Perot Interferometers in series. Total transmission contains only one spectral band.

Figure 8. Operation of cascaded FPI. The transmission peaks are aligned, thus only one band remains.

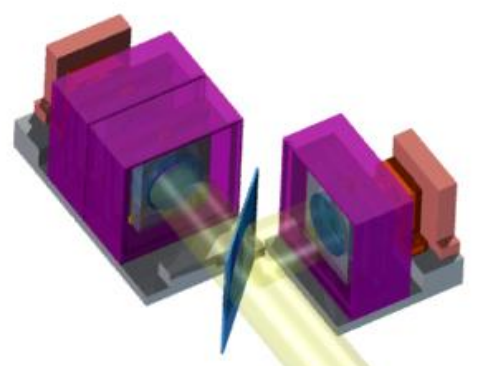

Figure 9. 3D model of the MIR channel of MIRMIS instrument. A dichroic beamsplitter is used to divide the light to different FPI's and detectors.

To further extend the operational range, the MIR channel is divided to two sub-channels, MIR1 $(2.5-4.0 \mu \mathrm{m})$ and

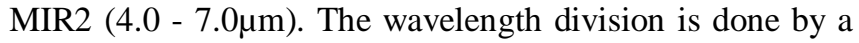
dichroic beamsplitter. The MIR channel is implemented as a single point spectrometer to reduce complexity.

\section{$M-A R G O$ [10]}

M-ARGO (Miniaturized Asteroid Remote Geophysical Observer) is an ESA technology demonstrator mission of a stand-alone 12U CubeSat mission to a small near-Earth asteroid. Primary payload is envisioned to be a NIR-MIR hyperspectral imager to map the composition of the target in high-resolution including hydration and organic compounds. This instrument is a combination from the instruments used in APEX and Comet Interceptor. The planned launch date for M-ARGO is in 2023.

\section{PICASSO - Stratospheric ozone measuring CubeSat [3]}

The PICo-satellite for Atmospheric and Space Science Observations (PICASSO) is an ESA project led by the Belgian Institute for Space Aeronomy. VTT has developed one of the payloads, VISION, for the mission. VISION has two scientific objectives: to study the ozone distribution in the stratosphere and to measure the atmospheric temperature profile up to the mesosphere. VISION is a spectral imager operating between $430-800 \mathrm{~nm}$, and it is capable of taking 2D snapshots of the sun at freely selectable wavelengths.

VISION instrument will observe the atmospheric limb during orbital Sun occultation. By addressing the radiation absorption in the Chappuis band for different tangent altitudes, the vertical profile of ozone can be retrieved. The mission is planned for launch in 2020 .

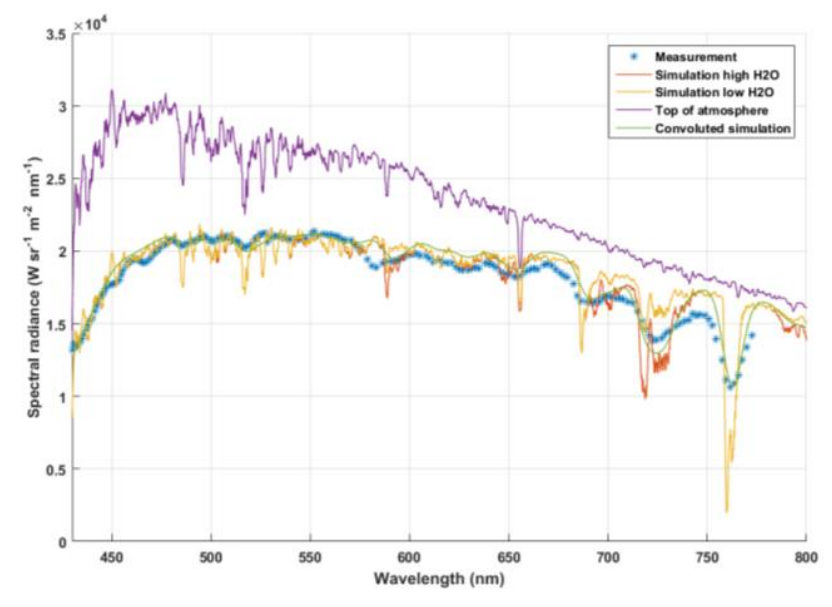

Figure 10. Ground based measurement of the atmospheric spectrum, measured with the engineering model of VISION. Simulation results are also shown for comparison. The main result is that VISION can detect the different gases (e.g. $\mathrm{H}_{2} \mathrm{O}, \mathrm{O}_{2}$ ) in the atmosphere, thus validating the instrument concept for this type of measurement.

\section{DEMONSTRATOR FLight RESULTS}

\section{Aalto-1 nanosatellite mission [11]}

The first demonstration of the tunable FPI -based spectral imagers in space was done on-board the Aalto-1 nanosatellite. Aalto-1 is the first Finnish nanosatellite, and it was successfully launched in June 2017. The main payload was Aalto-1 Spectral Imager (AaSI), was built by VTT. After the satellite was successfully detumbled in the spring 2018, the first cloud free images were acquired in August above Africa (Figure 11).

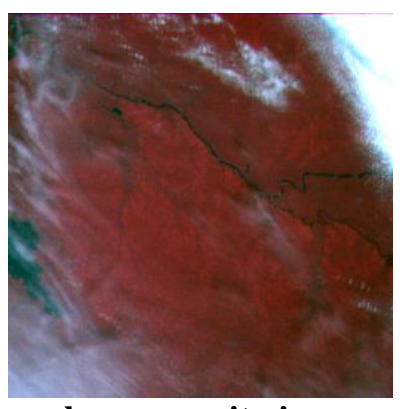

Figure 11. False-color composite image created with the spectral bands $565 \mathrm{~nm}(B), 670 \mathrm{~nm}(G)$ and $750 \mathrm{~nm}(R)$. 6 wavelength bands were measured in total. The image area is roughly $100 \mathrm{~km} \times 100 \mathrm{~km}$. 
This image verifies the functionality of the spectral imager, as the forest becomes highly reflective at wavelengths above $680 \mathrm{~nm}$ (red edge). The objective of this mission was to verify the operation of FPI-based spectral imager in space, and that was achieved.

\section{Reaktor Hello World nanosatellite mission}

The second demonstrator mission was the NIR spectral imager $(0.8-1.4 \mu \mathrm{m})$ on board the Reaktor Hello World nanosatellite. This satellite was the first commercial nanosatellite in Finland, a $2 \mathrm{U}$ CubeSat, built by Reaktor Space Lab Ltd. The payload was the NIR spectral imager as a precursor to the Hera mission. This was the first hyperspectral imager in a nanosatellite operating in wavelengths beyond $1.0 \mu \mathrm{m}$.

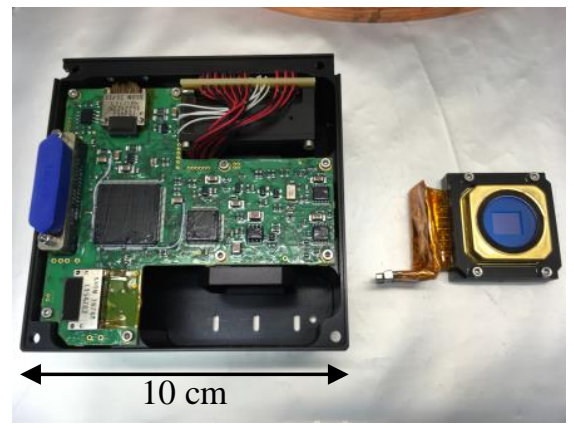

Figure 12. The flight model of the Hello World Spectral Imager before integration. On the right is the InGaAs detector array.

The satellite was successfully launched in November 2018, and the first images were acquired a few days later. Below are some example images showing different use cases, like $\mathrm{O}_{2}$ airglow emission in the upper atmosphere (i.e. detection and measurement of atmospheric gases) and land classification in Pakistan (i.e. differentiation of cultivated / non-cultivated land).

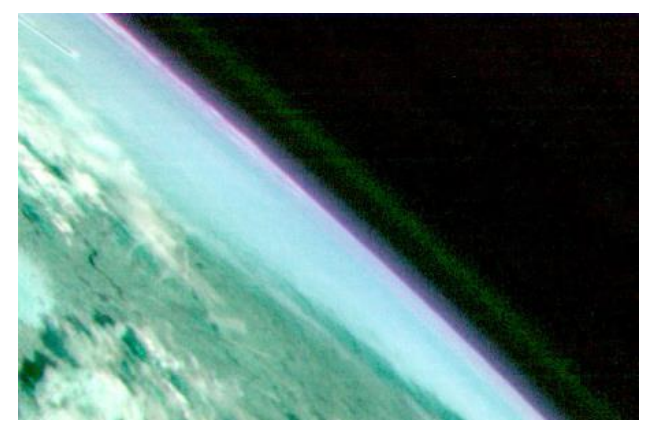

Figure 13. False color composite highlighting the $\mathrm{O} 2$ airglow at $1270 \mathrm{~nm}$ (the faint green band above horizon).

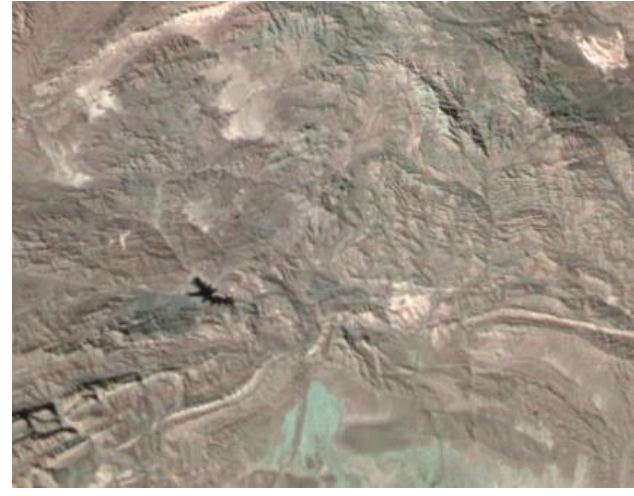

Figure 14. False color composite $(R=1310 \mathrm{~nm}, \mathrm{G}=1075$ $\mathrm{nm}, \mathrm{B}=1025 \mathrm{~nm}$ ) showing different types of land. The image is taken near Peshawar in Pakistan.

The Hello World mission has fulfilled all of its mission objectives, and it has proven that the technology can be adapted for infrared wavelengths for space applications.

\section{SUMMARY AND CONCLUSIONS}

The miniaturized spectral imaging developed at VTT is a novel way to construct spectral imagers. The technology allows very compact and robust construction, while still retaining good performance. The technology is also easily modified for different wavelength ranges, which make it possible to use the same basic design to many applications.

This technology has already been flight proven (twice), and it is proposed for use in many candidate instruments for upcoming exploration missions which require small and compact instruments.

Hopefully the technology will be demonstrated in deep space missions in the near future, which can open up possibilities to completely new applications e.g. drones in planetary atmospheres or miniaturized rovers/landers. 


\section{REFERENCES}

[1] Michel, P. et al. "European component of the AIDA mission to a binary asteroid: Characterization and interpretation of the impact of the DART mission", Advances in Space Research, vol. 62. issue 8, pp. 22612272,2018

[2] Kohout, T., Näsilä, A., Tikka, T. et al. "Feasibility of asteroid exploration using CubeSats - ASPECT case study", Advances in Space Research, vol. 62, issue 8, pp. 2239-2244, 2018

[3] Saari, H., Näsilä, A. et al. "Visible spectral imager for occultation and nightlow (VISION) for the PICASSO Mission", Proceedings of SPIE, vol. 9639, art.no. 96391M, 2015

[4] Saari, H. et al. "Novel miniaturized hyperspectral sensor for UAV and space applications", Proceedings of SPIE, vol. 7474, art.no. 74741M, 2009

[5] Fabry, C. and Perot, A. "Sur les Franges des Lames Minces Argentées et Leur Application à la Mesure de Petites Épaisseurs d'Air", Annales De Chimie et de Physique, vol. 12, pp. 459-501, 1897.

[6] Hecht, E. \& Zajac, A. "Optics", $1^{\text {st }}$ edition, AddisonWesley, 1974

[7] Küppers, M. et al. "HERA - The European component of the Asteroid impact deflection assessment (AIDA)", Proceedings of the International Astronautical Congress IAC, vol 2018-October, 2018

[8] Andrews, D., Wahlund, J-E, Kohout, T., and Penttilä, A. "Asteroid Prospection Explorer (APEX) Cubesat for the ESA Hera mission", Conference presentation at EPSC, EPSC-DPS2019-128, 2019

[9] Jones, G. and Snodgrass, C. "Comet Interceptor: A proposed ESA Mission to a Dynamically New Comet", Conference presentation at EPSC, EPSC-DPS2019-1679, 2019

[10] Walker, R. et al. "Miniaturized Asteroid Remote Geophysical Observer (M-ARGO): A stand-alone deep space CubeSat system for low cost science and exploration missions", IPPW Small Sat Short Course, Boulder, CO, USA, June 1028

[11] Kestilä, A., Tikka, T., Peitso, P., Rantanen, J., Näsilä, A., Nordling, K. et al. "Aalto-1 nanosatellite - technical description and mission objectives", Geoscientific Instrumentation Methods and Data Systems, vol. 2, issue 1,2013

\section{BIOGRAPHY}

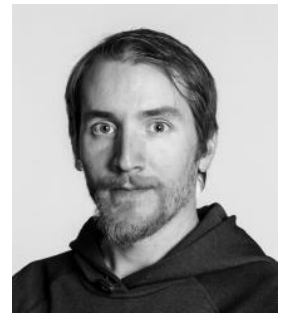

Antti Näsilä received a M.Sc. in Space Technology from Aalto Univeristy, Helsinki in 2013. He has been working at VTT since 2012, first as trainee, research scientist and senior scientist. Now he is working as the Research Team Leader for the Microspectrometers team, which specializes in developing miniaturized spectral measurement systems.

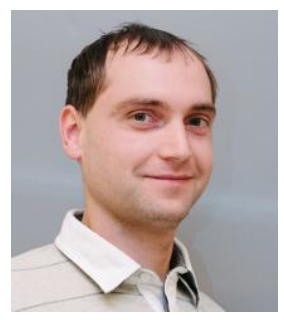

Dr. Tomas Kohout is an Academy Research Fellow at the University of Helsinki, Finland. He received a Ph.D.in geophysics from the University of Helsinki in 2009. He is an expert in planetary material compositions, physical properties, impact processes, and reflectance spectroscopy. Asteroid (14351) Tomaskohout is named after him. 